\title{
Findings from the 2017 Yearbook Section on Health Information Management
}

\author{
M. Bloomrosen', E. S. Berner², Section Editors for the IMIA Yearbook Section on Health \\ Information Management \\ ' Senior Director, Federal Affairs, Premier healthcare alliance, Washington, D.C., USA \\ ${ }_{2}^{2}$ Graduate Programs in Health Informatics, Department of Health Services Administration, \\ University of Alabama at Birmingham, Birmingham, AL, USA
}

\begin{abstract}
Summary
Objectives: To summarize the recent literature and research and present a selection of the best papers published in 2016 in the field of Health Information Management (HIM).

Methods: A systematic review of the literature for the IMIA Yearbook HIM section was performed by the two section editors with the help of a medical librarian. We searched bibliographic databases for HIM-related papers were searched using both MeSH headings and key words in titles and abstracts. A shortlist of candidate best papers was first selected by section editors before being peer-reviewed by independent external reviewers. Results: The five papers selected as 'Best Papers' illustrate a variety of themes, include authors from a variety of countries, and were published in some of the best journals in the field. The themes of the five best papers include health information exchange, personal health records, patient engagement, data quality, and e-quality measures.

Conclusions: The discipline of Health Information Management is increasingly becoming allied with the field of Biomedical Informatics in that both disciplines have interests in common. Traditional HIM areas of expertise (in the pre-electronic health record world), such as coding and privacy and security of health information, are necessary for the electronic exchange and secondary use of health information. With the changes in healthcare delivery brought by the use of electronic health records, addressing issues of information governance is essential. This synopsis discusses these key issues at the intersection of HIM and informatics, examines the potential challenges, and points the way for best practices, future research, and public policy considerations and directions.
\end{abstract}

\section{Keywords}

Health Information Management; health information exchange; personal health records; health informatics; secondary data use

Yearb Med Inform 2017:78-83

http://dx.doi.org/10.15265//Y-2017-025

Published online September 11, 2017

\section{Introduction}

In the era of paper-based medical records, the disciplines of Health Information Management (HIM) and medical, clinical and/or health informatics were typically quite separate, each focusing on different, albeit complementary, domains and skills. However, since the discussions around the issues of privacy, confidentiality, and security of Electronic Health Records (EHRs) that began in the 1990s in the United States (U.S.) with the passage of the Health Insurance Portability and Accountability Act of 1996 (HIPAA) legislation, the interests of these disciplines have become increasingly aligned $[1,2]$. Privacy of medical records and release of health information have traditionally been an HIM focus; additionally, HIM has assumed over the years roles and responsibilities in other domains, including health Information Technology (health IT) and data governance, and personal health records (PHRs). Similarly, digitization of health data and information, data sharing and linking, and adoption and implementation of EHRs, PHRs, and Health Information Exchanges (HIEs) have introduced new considerations about health informatics practice, research, and innovation [3, 4]. Issues regarding training, education, skills, and competencies across the health information management and health informatics continuum have also been explored [5-8].

In addition, HIM's traditional expertise in classification systems and coding of clinical data is particularly relevant to the issues of standardization for secondary use of clinical data, the theme of the 2017 IMIA Yearbook, as well as Health Information Exchange
(HIE) more broadly. Now that EHRs have been more widely adopted $[9,10]$, HIM can play a crucial role in the growing interest in health data analytics, population health, and patient engagement in health care, research, and outcomes. Hence, it is fitting that the 2017 IMIA Yearbook includes a new section focused on HIM.

While the articles included in this section exemplify many of the HIM themes alluded to above, they also fit well within traditional informatics themes, further illustrating the increasing synergy between the two disciplines. Below, we describe our method for identifying and evaluating key HIM articles published in 2016, the themes that they illustrate, and how we selected the five best papers, which we summarize at the end of this synopsis.

\section{Methods}

At the end of December 2016, with the assistance of a medical librarian, the editors of the HIM section conducted a search of both PubMed and Embase using both MeSH headings and keywords in the titles and abstracts for Health Information Management. The publication year was 2016 and did not include those articles that were e-published ahead of print. The query for PubMed was: "Health Information Management"[Mesh] OR "Health Information Management" [tiab] OR "HIM J"[Journal] OR "JAHIMA"[Journal]", which yielded 159 results. The query for EMBASE was "medical information system"/exp/mj OR "health information management":ti,ab OR 
"clinical information system":ti,ab OR "clinical pharmacy information systems":ti,ab OR "Health Information Exchange":ti,ab OR "health information management":ti,ab OR "health information manager":ti,ab OR "health information network":ti,ab OR "health information system":ti,ab OR "health information systems":ti,ab OR "IS-H med":ti,ab OR "medical information service":ti,ab OR "Health Information Management Journal", which, after eliminating duplicates that had appeared in the PubMed search yielded 423 articles.

The 582 unique articles were rated by both section editors, who generally excluded articles that were opinion pieces or editorials, non-English articles, or articles where the full text of the article was not readily available. Each of the two section editors judged the relevance and quality of the articles independently. Those that both co-editors rated as not appropriate were excluded automatically. The rest of the articles were discussed and disagreements adjudicated to arrive at 15 articles that, based primarily on the abstracts, were judged to be appropriate and of good quality. The full texts of these 15 articles were then rated independently by both section editors, one of the Yearbook editors, and at least one external peer reviewer.

The five 'Best Papers' were those whose average rank was the highest, and with only one exception, were rated positively by all reviewers. These papers illustrate a variety of themes, include authors from a variety of countries, and were published in some of the best journals in the field. The themes of the five best papers include Health Information Exchange, personal health records, patient engagement, data quality, and e-quality measures.

The survey paper of the 2017 HIM section [11] focuses on a different perspective and describes the impact on the practice and profession of HIM of the issues related to data governance, new terminology standards, and data analytics. Noting the evolution of patient medical records from paper to electronic media and the accessibility of new data sources and uses, the paper focuses on four areas: 1) the increasing need for effective data and information governance; 2) the development of a terminology standards certification; 3 ) the upcoming release and implementation of the International Classification of Diseases, 11th edition (ICD-11); and 4) the growing role of data analytics within HIM. These issues influence the effective use of clinical information and data for research, population and public health, quality improvement, and other secondary uses of clinical information. The paper's conclusion is that because of the digitization of health records and the expanding amount, complexity, and secondary uses of patient data, HIM practitioners require skills in leadership, data, and informatics in addition to traditional health information science and coding skills.

Table 1 Best paper selection of articles for the IMIA Yearbook of Medical Informatics 2017 in the section 'Health Information Management'. The articles are listed in alphabetical order of the first author's surname.

Section

Health Information Management

- Bahous MC, Shadmi E. Health information exchange and information gaps in referrals to a pediatric emergency department. Int J Med Inform 2016;87:68-74.

- Esmaeilzadeh P, Sambasivan M. Health Information Exchange (HIE): A literature review, assimilation pattern and a proposed classification for a new policy approach. J Biomed Inform 2016;64:74-86.

- Massoudi BL, Marcial LH, Tant E, Adler-Milstein J, West SL. Using health information exchanges to calculate clinical quality measures: A study of barriers and facilitators. Healthc (Amst) 2016;4(2):104-8.

- Toscos T, Daley C, Heral L, Doshi R, Chen YC, Eckert GJ, Plant RL, Mirro MJ. Impact of electronic personal health record use on engagement and intermediate health outcomes among cardiac patients: a quasi-experimental study. J Am Med Inform Assoc 2016;23(1):119-28.

- Warner JL, Rioth MJ, Mandl KD, Mandel JC, Kreda DA, Kohane IS, Carbone D, Oreto R, Wang L, Zhu S, Yao H, Alterovitz G. SMART precision cancer medicine: a FHIR-based app to provide genomic information at the point of care. J Am Med Inform Assoc 2016;23(4):701-10.

The lead author of the survey paper of the 2017 HIM section [11], Susan Fenton, PhD, RHIA, FAHIMA, was selected for her expertise in both HIM and Informatics and her co-authors bring an international perspective as well. Together, the survey paper of the 2017 HIM section and the 15 candidate best papers published in 2016 provide a picture of the current state of the intersection of the disciplines of health information management and informatics.

\section{Results}

We discuss the 15 reviewed articles under four broad themes, although several of them span these or other themes. The four themes are (1) Health Information Exchange, (2) Data standards, (3) Data quality for secondary use of EHR data (data organization, quality, accuracy, completeness), and (4) Personal Health Records, Personal Health Information Management, and Patient Engagement. These themes, and the articles supporting them, present a picture of major foci in the HIM and Informatics landscape today.

\section{Health Information Exchange}

Health Information Exchange (HIE) is a key concern for HIM, both for clinical information exchange among providers and for secondary use of data. HIE has become more prevalent as the use of EHRs has expanded and it promises to be used even more in the future. While several of the articles in other sections also relate to HIE, those in this section focus on the process of exchanging information for clinical use among providers caring for a given patient as well as the entities conducting the exchange. HIE has been attempted for years, but there have been several challenges. The systematic review by Eden and colleagues address the barriers and facilitators of HIE, expanding research done in the US. to an international context [12]. This article is an expansion of an Evidence Report/Technology Assessment on HIE funded by the U.S. Agency for Healthcare 
Research and Quality [13]. Eden and colleagues found that there were similarities and differences in the barriers identified from US sites and those outside the US. They identified three categories of barriers: "completeness of information, organization and workflow, and technology and user needs". As with other health IT interventions, the authors found that consideration of the workflow and involvement of providers were more likely to lead to success. They also found that, in the US, completeness of information was often a major barrier, but this issue was not seen as problematic outside the US.

Bahous and Shadmi's study [14] casts more light on the issue of the completeness of information. They examined the completeness of three different sources of information (HIE, referral letter, and medical history from the patient) and found that while some information was common to each source, there were also gaps in each, and these gaps were not the same, so that the three sources of information were complementary. This study was selected as a best paper and is described in more detail at the end of this synopsis.

The paper from Esmaeilzadeh and Sambasivan [15], also designated as a best paper and described in more detail below, takes the review of the literature on HIE a step further and identifies stages of adoption and assimilation of HIE. These stages are important for administrators who are contemplating establishing Health Information Exchanges so that they can plan adequately for their involvement.

The involvement of top leadership, like considering the workflow and involving the clinicians in planning, is crucial for the success of any implementation of health information technology including HIE. Vest and Kash conducted a qualitative study of hospital administrators and found, interestingly, that many hospitals were more motivated to institute enterprise-wide exchanges, rather than larger community-wide exchanges [16]. This set of studies provides an important summary of the current state of HIE. Many of the barriers identified are attitudinal, organizational, and behavioral. However, one of the main technical barriers is the challenge of appropriate standards for information exchange.

\section{Standards}

A key barrier to the exchange of information is arriving at agreement on standards, both on terminology standards, traditionally within the domain of HIM and addressed by Fenton et al. [11], and on messaging standards. Three of the 15 candidate papers address this issue, one of which was selected as a best paper. This paper, by Warner and colleagues [17] describes an application in oncology using the new Fast Healthcare Interoperability Resources (FHIR), which they also describe. FHIR has received a great deal of attention and is currently an HL7 Standard for Trial Use [18]. Major EHR vendors have expressed interest in FHIR which bodes well for promoting interoperability and Health Information Exchange. The other two articles compare different standards. Warzel and Reeves [19] compared different content standards and Metroka et al. [20] compared the quality of data uploaded to an immunization information system using HL7, manual data entry, and the upload of an EHR data file. They found that HL 7 was both more complete and more timely than other methods of uploading information. As these electronic standards for data exchange continue to evolve, their use will facilitate Health Information Exchange for clinical purposes as well as secondary use of clinical data for other purposes. In the next section, we describe a set of papers that examine the data quality, completeness, and organization of EHR data for secondary use.

\section{Secondary Use of EHR Data}

Appropriate secondary use of EHR data depends on the quality and organization of the primary EHR data. Quality of data is usually considered to include dimensions of accuracy, completeness, and timeliness. The adequacy of the data for a given purpose depends on the particular purpose. For instance, Batra and Sachdeva have developed approaches to organizing EHR data for data mining [21]. However, if data is not accurate and complete, organization alone will not help. Gray and Finch examined the completeness of narrative and coded data for an injury surveillance system and found completeness problems in both kinds of data [22]. Addressing those problems is not easy. Ghosh, McCarthy, and Halcomb found that the quality of the data needed for population health planning should be improved and they designed an intervention to address the problems [23]. They found that behavioral issues needed to be addressed to assure that primary data was sufficiently complete. Massoudi and colleagues conducted a qualitative study to examine the perceptions of the quality of data available through Health Information Exchange for documenting electronic clinical quality measures [24]. They found problems in data quality as well as other issues that make computing these measures challenging. The Massoudi et al. paper was selected as a best paper and is described in more detail below. As the use of EHRs increases, there is more and more interest in secondary use of EHR data. As these studies demonstrate, there are still challenges in terms of data quality, completeness, and organization of EHR data.

\section{Personal Health Information Management}

Another area that has been a focus of HIM is the area of personal health information management, often using PHRs or patient portals where the health record is tethered to the health system EHR. One of the main expectations of the use of PHRs is that they can promote patient engagement and improve health outcomes. The articles described below examined patient engagement, the use of personal health records, and the relationship between the two. Camuso and colleagues conducted a literature review examining tools and methods to engage patients in the development of patient-reported outcome measures in cancer [25]. They found that often patients were engaged very late in the process. Kim and Abner examined skills associated with effective use of PHRs [26]. They identified a variety of skills including both collecting 
and sharing information. They found that a patient's health knowledge was related to collecting information. Hasman, Rapp, and Brown discussed the effective use of a home-based record for developing countries [27]. The final paper in this section was designated as one of the best papers and is discussed in detail below. In this paper, Toscos and colleagues tested the assumption that PHR use would lead to increased patient engagement in their health care [28]. Their conclusion that "PHR use had minimal impact on intermediate health outcomes and no significant impact on patient engagement" raises questions about the rationale for PHR use. While there may be many good reasons to promote PHR use, the Toscos et al. study reminds us that we must test our assumptions about their potential for impacting patient outcomes.

\section{Conclusion}

The discipline of Health Information Management increasingly is becoming allied with the field of medical and health Informatics in that both disciplines have interests in common and traditional HIM areas of expertise in the pre-electronic health world, such as coding and privacy and security of health information, are necessary for the electronic exchange and secondary use of health information. With the changes in healthcare delivery brought about by the use of electronic health records, addressing issues of information governance is essential. This synopsis discusses these key issues at the intersection of HIM and informatics, examines the challenges, and points the way for best practices and future research directions.

\section{Acknowledgement}

We would like to acknowledge Megan Bell, who assisted us with formulating our search strategy. We also appreciate the guidance and support of the entire Yearbook editorial team, especially Brigitte Séroussi, Lina Soualmia, Adrien Ugon, and Martina Hutter, as well as the reviewers who participated in the selection process of the best papers.

\section{References}

1. Health insurance portability and accountability act of 1996, Pub. L. No. 104-191;1996.

2. University of Alabama at Birmingham. Component 5, Unit 15, History of Health IT Organizations. Health IT Curriculum: Office of the National Coordinator for Health Information Technology; 2013.

3. Open Notes [Available from: https://www.opennotes.org.] Accessed May 30, 2017.

4. Atun R, Lussier Y, Poon C, Wong STC, Yang G-Z. Big Data for Health. IEEE J Biomed Health Inform. [Internet]. July 8, 2015. Available from: http://jbhi.embs.org/2015/07/08/special-issue-bigdata-for-health. Accessed May 30, 2017.

5. Dorsey AD, Clements K, Garrie RL, Houser SH, Berner ES. Bridging the gap: A collaborative approach to health information management and informatics education. Appl Clin Inform 2015;6(2):211-23.

6. Joint Work Force Task Force. Health information management and informatics core competencies for individuals working with electronic health records; 2008.[Available from: https://www.amia. org/sites/default/files/Joint-Work-Force-TaskForce-2008.pdf ]. Accessed May 30, 2017.

7. Gibson CJ, Dixon BE, Abrams K. Convergent evolution of health information management and health informatics: a perspective on the future of information professionals in health care. Appl Clin Inform 2015;6(1):163-84.

8. Garde S, Harrison D, Huque M, Hovenga EJ. Building health informatics skills for health professionals: results from the Australian Health Informatics Skill Needs Survey. Aust Health Rev 2006;30(1):34-45.

9. Office of the National Coordinator for Health Information Technology. Office-based physician electronic health record adoption. Health IT Quick Stat \#50; December 2016. [Available from: http://dashboard.healthit.gov/quickstats/pages/ physician-ehr-adoption-trends.php] Accessed May 30, 2017.

10. Office of the National Coordinator for Health Information Technology. Non-federal acute care hospital electronic health record adoption. Health IT Quick-Stat \#47; May 2016. [Available from: http://dashboard.healthit.gov/quickstats/pages/ FIG-Hospital-EHR-Adoption.php ] Accessed May 30, 2017.

11. Fenton SH, Low S, Abrams K, Butler-Henderson $\mathrm{K}$. Health information management: changing with time. Yearb Med Inform 2017. To appear.

12. Eden KB, Totten AM, Kassakian SZ, Gorman PN, McDonagh MS, Devine B, et al. Barriers and facilitators to exchanging health information: a systematic review. Int J Med Inform 2016;88:44-51.

13. Hersh W, Totten A, Eden K, Devine B, Gorman P, Kassakian S, et al. Health Information Exchange. Evidence Report/Technology Assessment No. 220. Rockville, MD: Pacific Northwest Evidence-based Practice Center; 2015. [Available from: https://effectivehealthcare.ahrq.gov/ehc/products/572/2154/ health-information-exchange-report-151201.pdf] Accessed May 30, 2017.
14. Bahous MC, Shadmi E. Health Information Exchange and information gaps in referrals to a pediatric emergency department. Int J Med Inform 2016;87:68-74.

15. Esmaeilzadeh P, Sambasivan M. Health Information Exchange (HIE): A literature review, assimilation pattern and a proposed classification for a new policy approach. J Biomed Inform 2016;64:74-86.

16. Vest JR, Kash BA. Differing strategies to meet information-sharing needs: publicly supported community Health Information Exchanges versus health systems' enterprise Health Information Exchanges. Milbank Q 2016;94(1):77-108.

17. Warner JL, Rioth MJ, Mandl KD, Mandel JC, Kreda DA, Kohane IS, et al. SMART precision cancer medicine: a FHIR-based app to provide genomic information at the point of care. J Am Med Inform Assoc 2016;23(4):701-10.

18. Welcome to FHIR: HL7 International; [Available from: http://hl7.org/fhir/index.html] Accessed May 30, 2017

19. Warzel DB, Reeves DM. Cross-walking health content standards using the ISO/IEC 11179 metadata registries standard. J AHIMA 2016;87(7):46-9.

20. Metroka AE, Papadouka V, Ternier A, Zucker JR. Effects of Health Level 7 messaging on data quality in New York City's immunization information xystem, 2014. Public Health Rep 2016;131(4):583-7.

21. Batra S, Sachdeva S. Organizing standardized electronic healthcare records data for mining. Health Policy Technol 2016;5:226-42.

22. Gray SE, Finch CF. Assessing the completeness of coded and narrative data from the Victorian Emergency Minimum Dataset using injuries sustained during fitness activities as a case study. BMC Emerg Med 2016;16(1):24.

23. Ghosh A, McCarthy S, Halcomb E. Perceptions of primary care staff on a regional data quality intervention in Australian general practice: a qualitative study. BMC Fam Pract 2016;17:50.

24. Massoudi BL, Marcial LH, Tant E, Adler-Milstein J, West SL. Using Health Information Exchanges to calculate clinical quality measures: A study of barriers and facilitators. Healthc (Amst) 2016;4(2):104-8

25. Camuso N, Bajaj P, Dudgeon D, Mitera G. Engaging patients as partners in developing patient-reported outcome measures in cancer - a review of the literature. Support Care Cancer 2016;24(8):3543-9.

26. Kim S, Abner E. Predictors affecting personal health information management skills. Inform Health Soc Care 2016;41(3):211-29.

27. Hasman A, Rapp A, Brown DW. Revitalizing the home-based record: Reflections from an innovative south-south exchange for optimizing the quality, availability and use of home-based records in immunization systems. Vaccine 2016;34(47):5697-9.

28. Toscos T, Daley C, Heral L, Doshi R, Chen YC, Eckert GJ, et al. Impact of electronic personal health record use on engagement and intermediate health outcomes among cardiac patients: a quasi-experimental study. J Am Med Inform Assoc 2016;23(1):119-28.

29. Health Information and Management Systems Society (HIMSS). HIMSS Health IT Resource Library: Health Information Exchange: HIMSS; [Available from: http://www.himss.org/library/health-informa- 
tion-exchange] Accessed May 30, 2017.

30. Adler-Milstein J, Bates DW, Jha AK. A survey of Health Information Exchange organizations in the United States: implications for meaningful use. Ann Intern Med 2011;154(10):666-71.

31. SMART: An App Platform for Healthcare [Available from: https://smarthealthit.org] Accessed May 30, 2017.

Correspondence to:

Meryl Bloomrosen

E-mail: Meryl_Bloomrosen@premierinc.com

Appendix: Content Summaries of Selected Best Papers for the IMIA Yearbook 2017, Section Health Information Management

\section{Bahous MC, Shadmi E \\ Health Information Exchange and information gaps in referrals to a pediatric emergency department}

\section{Int J Med Inform 2016;87:68-74}

This paper assesses the extent of information congruence and/or gaps among three information sources available at admission to a pediatric Emergency Department (ED): a Health Information Exchange (HIE) system; physicians' referral letters; and information collected directly from patients/parents. The authors conducted a retrospective cohort study of 170 medical records of children aged 6 months to 18 years referred to an Israeli pediatric ED. Each medical record was reviewed for the presence of key pieces of information in each of the information sources. The results demonstrated that the most informative source was the patient's medical history; next was the HIE system; the least informative was the referral letter.

The authors' analyses indicate that each of the information sources available lacks important data and each makes its own unique contribution. Further, the authors conclude that improving documentation in electronic health records can address significant information gaps in HIE. With the increasing diffusion of electronic health records and HIE, the availability, completeness, accuracy and quality of data, and documentation available at the point of care continues to be a major issue that must be addressed.

\section{Esmaeilzadeh P, Sambasivan M \\ Health Information Exchange (HIE): A literature review, assimilation pattern and a proposed classification for a new policy approach}

\section{J Biomed Inform 2016;64:74-86}

The authors conducted a study to define Health Information Exchange (HIE) assimilation phases, and they proposed a classification to highlight the unique issues associated with these phases. These authors define HIE as the electronic transfer of patient data and health information between healthcare providers. The study includes a literature review of existing studies of HIE between January 2005 and April 2016. Out of an initial 254 articles, 44 studies met the inclusion criteria and were reviewed. The paper discusses the authors' use of the term "assimilation" rather than "adoption", noting that although the two terms are often used interchangeably, there has been no consensus on the definition of adoption. The authors emphasize that they use assimilation to better articulate the complex nature of HIE and all related factors affecting HIE at various levels of analysis. The authors describe four main phases of HIE assimilation: initiation, organizational adoption decision, implementation, and institutionalization. The authors note that most of the existing studies focused on only one HIE assimilation phase --- institutionalization. The paper shows the importance of raising national awareness of HIE potential benefits, financial incentive programs, use of standard guidelines, implementation of certified technology, technical assistance, training programs, and trust between healthcare providers.

\section{Massoudi BL, Marcial LH, Tant E, Adler- Milstein J, West SL \\ Using Health Information Exchanges to calculate clinical quality measures: A study of barriers and facilitators}

Healthc (Amst) 2016;4(2):104-8

The study explores the extent to which Health Information Exchanges (defined as organizations dedicated to the secure exchange of health-related data [29]) are used to calculate clinical quality measures. The authors' goals were to understand the availability of EHR data needed to compute clinical quality measures (CQMs), and the barriers to data collection and/or CQM calculation. The particular CQMs examined were the National Quality Forum (NQF) eMeasures for the ABCs (aspirin therapy, blood pressure screening, cholesterol screening, and smoking cessation), body mass index (BMI), and diabetes measures.

The authors conducted semi-structured interviews with 36 Health Information Exchanges that, in a prior national HIE survey [30], self-reported their ability to exchange data that could be used to calculate standard quality metrics. Identified barriers to computing CQMs include: agreements about access to information and data sharing; costs; limited EHR functionality and availability of EHR vendor services. Specific challenges include data accessibility, usability, and formatting; lack of EHR data standardization; diverse HIE business and governance models; potential purposes of HIE data (re) use; and the business case/value proposition for HIEs. The authors' conclusions present suggestions for future policy.

\section{Toscos T, Daley C, Heral L, Doshi R, Chen YC, Eckert GJ, Plant RL, Mirro MJ} Impact of electronic personal health record use on engagement and intermediate health outcomes among cardiac patients: a quasi-experimental study

\section{J Am Med Inform Assoc 2016;23(1):119-28}

This article addresses issues related to the effect of Personal Health Records (PHRs) on patient engagement and patient outcomes. The study was conducted to determine the impact of tethered PHR use on patient engagement and intermediate health outcomes among patients with coronary artery disease (CAD). For the purposes of this effort, a PHR was defined "as an Internet-based set of tools that allows people to access and coordinate their lifelong health information and make appropriate parts of it available to 
those who need it." The authors evaluated patient engagement using the 13 -item Patient Activation Measure $\AA$ (PAM $\left.{ }^{\circledR}\right)$, a validated measure of patient activation. They also measured health outcomes (smoking cessation, weight control, diet, exercise) which may be influenced by patient engagement. The researchers used a quasi-experimental design with a single group involving pre-post evaluations. The authors found that PHR use did not impact patient engagement and only had a limited impact on health outcomes. The article sheds light on evolving issues related to patients' engagement in managing their chronic conditions; patients' role in their health outcomes; the use of technologies to improve health outcomes; and the various sociotechnical factors affecting health information and informatics research.
Warner JL, Rioth MJ, Mandl KD, Mandel JC, Kreda DA, Kohane IS, Carbone D, Oreto R, Wang L, Zhu S, Yao H, Alterovitz G

SMART precision cancer medicine: a FHIRbased app to provide genomic information at the point of care

\section{J Am Med Inform Assoc 2016;23(4):701-10}

The article discusses precision cancer medicine which increasingly requires access to genomic data within the clinical workflow and tools to assist clinical interpretation and enable decisions. The authors note that since most electronic health record (EHR) systems do not offer such functionality, they developed an EHR-agnostic clinico-genomic mobile application (app) to improve point-of-care decisions. The authors developed an opensource application based on the Substitutable
Medical Applications and Reusable Technology (SMART) Health IT platform [31], an open-access Application Programming Interface (API) that enables apps to run broadly across the health care ecosystem. They used HL7's FHIR standard and its extension features to represent molecular profile data. The app presents population-level genomic health information to oncologists and their patients in real time during clinical practice. The article describes the application development, pilot testing, samples and screens. The source code for the app has been made openly available. Results from the project include descriptions of clinician feedback about the app. The article contributes to the understanding of the use of mobile technologies and applications in cancer diagnosis, care, and treatment while exploring the evolving nature of precision medicine and the use of new interoperability standards. 